\title{
Convergence of computing, communication, and caching in internet of things
}

\author{
Mohammed Amine Bouras, Fadi Farha, and Huansheng Ning*
}

\begin{abstract}
Internet of Things (loTs) is a big world of connected objects, including the small and low-resources devices, like sensors, as well as the full-functional computing devices, such as servers and routers in the core network. With the emerging of new loT-based applications, such as smart transportation, smart agriculture, healthcare, and others, there is a need for making great efforts to achieve a balance in using the loT resources, including Computing, Communication, and Caching. This paper provides an overview of the convergence of Computing, Communication, and Caching (CCC) by covering the loT technology trends. At first, we give a snapshot of technology trends in communication, computing, and caching. As well, we describe the convergence in sensors, devices, and gateways. Addressing the aspect of convergence, we discuss the relationship between CCC technologies in collecting, indexing, processing, and storing data in loT. Also, we introduce the three dimensions of the loTs based on CCC. We explore different existing technologies that help to solve bottlenecks caused by a large number of physical devices in loT. Finally, we propose future research directions and open problems in the convergence of communication, computing, and cashing with sensing and actuating devices.
\end{abstract}

Key words: convergence; computing; communication; caching; sensing; actuating; Internet of Things (loTs)

\section{Introduction}

The ubiquitously connected environment (i.e., Internet of Things (IoTs)) is a computing model in which all electronic appliances will be connected to the Internet and name themselves to other appliances; the concept is qualifying concrete objects to see, hear, think, and perform jobs by having them communicate together ${ }^{[1]}$. The IoT alters these bodies from being traditional to being smart by taking advantage of essential technologies, such as communication technologies, computing, and data management.

Information and Communication Technology (ICT) is

- Mohammed Amine Bouras, Fadi Farha, and Huansheng Ning are with the School of Computer and Communication Engineering, University of Science and Technology Beijing, Beijing 100083, China. E-mail: bouras.ma@xs.ustb.edu.cn; fadi_farha@xs.ustb.edu.cn; ninghuansheng@ustb.edu.cn.

* To whom correspondence should be addressed.

Manuscript received: 2020-02-17; accepted: 2020-03-05 the infrastructure that enables the modern computing model IoT. The components of ICT are different, but they share the same pillar technologies, such as communication, computing, and caching. Computing is defined as a process of utilizing hardware and software or a computer system to perform and complete specific tasks, such as sending an email or using a cell phone. Communication technology allows exchanging data and resources between communication nodes and connects devices and peoples. Caching refers to the temporal storing of the data at the local device. These data could then be transmitted to the server on-demand based on the delay tolerance scheme identified by the application scenarios.

Nowadays, there is no clear margin between the Computing, Communications, and Caching (CCC). However, the bandwidth of the network has come to a standstill, set side by side to the agile growing of data processing rate; for instance, about 5 Gigabyte data is produced by a Boeing 787 every second ${ }^{[2]}$. The 
bandwidth in the thick of the aircraft and satellite or base station is not sufficient for massive data transmission. Consider a self-driving car as another example, 1 Gigabyte is created by the car every second, and it necessitates real-time processing for the vehicle to make the correct decision ${ }^{[3]}$, if all data need to be sent, the response time would be too long; note that the present-day network bandwidth and reliability would be a confront for its capacity of backing a considerable number of automobiles in one territory. However, people are also producing data nowadays from their different devices (PC, mobile phone, smart watch, etc.), for example, it is a habit today that people watch and record videos, take and upload pictures to share them through SnapChat, Netflix, YouTube, or DropBox. Moreover, in every single minute, Netflix subscribers stream 994444 hours of video; Youtube users watch 4500000 videos, and Twitch users view 1000000 hours of videos; Instagram application clients upload 55140 new pictures and post 277777 stories $^{[4]}$ In this case, the emergent quantity of data generated from different devices and systems and the speed of data transmission are becoming bottlenecks.

IoT communication technologies connect different objects to deliver specific smart facilities. Examples about the communication protocols used in the IoT are Long Term Evolution (LTE)-Advanced, Wi-Fi, Bluetooth, IEEE 802.15.4, and Z-wave. As well Near Field Communication (NFC), Ultra-Wide Bandwidth (UWB) and RFID ${ }^{[1]}$ are adopted. Computing hardware items (e.g., microprocessors, microcontrollers ${ }^{[5]}$, and Field Programmable Gate Arrays (FPGAs) ${ }^{[6]}$ ) and software application characterize the computational aptitude of the IoT. Diverse hardware industrialized to run IoT applications, such as Arduino, UDOO, FriendlyARM, and Intel Galile, in addition to, numerous software platforms are operated to distribute IoT functionalities. Midst these platforms, operating systems are vital since they run for the entire life time of a device. There are several real-time operating systems (Contiki $^{[7]}$, Tiny OS ${ }^{[8]}$, Lite OS ${ }^{[9]}$, and Riot $\mathrm{OS}^{[10]}$ ).

IoT is also one of the domains in which data is amassed in a high speed and volume. Big data is an essential part of IoT in term of data management (collect, store, and process); big data is a large and complex dataset or stream which is hard to process using old-style database management tools or processing applications. The fast growth of sensing technology makes IoT data management more complicated, especially for the large amounts of unstructured data ${ }^{[11]}$. Cloud paradigm is an essential computational part of the IoT. These platforms provide facilities for smart objects to send their data to the cloud, for big data to be processed in real time, and ultimately for end-users to benefit from the knowledge extracted from the collected big data ${ }^{[1]}$

In this paper, we discuss the convergence of communication, computing, and caching and provide a review of technology convergence since 1977, the need for convergence, while also focusing on IoT ethics with the convergence.

The paper is organized as follows: Section 2 presents the related works on the convergence of CCC. Section 3 explores the communication and computing trends, assesses the cutting-edge technology, promised computing, and network technologies. Section 4 expresses the role of caching in communication and computing. Section 5 is about the convergence inside smart physical devices of IoT providing information about sensors, devices, and gateways. Section 5.3 illustrates the convergence of CCC in IoT, including the importance of convergence. In Sections 7 and 8, we conclude with several key issues, future work, and conclusion.

\section{Related work}

The convergence of communication and computing has been noted and remarked in the old days; certainly, the current interrelationship is more affluent than any time before due to the accelerated development of technology.

In March 1977, David Farber and Paul Baran investigated the convergence of computing and communication systems from the nature of new services, the importance to the economy, and the advanced technology. In particular, they discussed such convergence with a typical communication and computing company ${ }^{[12]}$.

Twenty years later, Messerschmitt ${ }^{[13]}$ and Decina 
and Trecordi $^{[14]}$ did a wide-ranging study, mainly from a technical perspective, on the convergence of computing and communication. Messerschmitt ${ }^{[13]}$ was reasonable about the future as the march of technology deemphasizes traditional performance metrics in favor of more functionality complex and numerous systems and applications. In their investigation, Decina and Trecordi ${ }^{[14]}$ described the convergence of the communication and computing industries to a common vision of networking models for an integrated service information infrastructure meeting the rigid requirements of multimedia application.

In 2012, Kim and Kumar ${ }^{[15]}$ defined Cyber-Physical Systems (CPSs) as the next generation of engineered systems in which computing, communication, and control technologies are strongly integrated. They also claimed that this emerging multidisciplinary edge will drive to an innovative way of human living.

In September 2016, $\operatorname{Fan}^{[16]}$ gave a snapshot of convergence of CCC. Also, he mentioned the role of storage to the communication and computing which was often omitted in previous works. Besides, he introduced a new concept called effective information system capacity.

\section{Communication and computing trend}

In the past few years, the frontiers among Communications Technology (CT), which encompasses electronic systems used for communication between individuals or groups and Information Technology (IT), which invokes to hardware and software utilized to store, restore, and process data, have become more and more identical. The swift consolidating of IT and CT is taking place at three rows of technology innovation cloud, pipe, and device ${ }^{[17]}$. In this section, we introduce the merging of IT and CT in some emerging technologies and give a brief introduction about the convergence of IT and CT.

\subsection{Developments trends of communication}

\subsubsection{G network}

Mobile devices have become an essential part of our quotidian lives, and so is the mobile network infrastructure that connects them which turns out to be more demanding. It is set to take on an even advanced aspect of the fifth Generation (5G) mobile systems envisioned backing a vast design of devices and services ${ }^{[18]}$. The major pivot is represented by the $5 \mathrm{G}$ cellular network architecture, gigantic divers input divers output technology, and Device-to-Device (D2D) communication $^{[19]}$.

Mobile and wireless networks have made remarkable development in the last few years. The revelation of the $5 \mathrm{G}$ wireless communications lies in providing very high data rates, extremely low latency, manifold increase in base station capacity, and significant upgrading in users' perceived Quality of Service (QoS), compared to the ongoing 4G LTE networks ${ }^{[20]}$.

With an epidemic growth in the ongoing mobile networks users, $4 \mathrm{G}$ will now be smoothly swapped for $5 \mathrm{G}$ with a leading connection technique named Beam Division Multiple Access (BDMA) ${ }^{[21]}$. The approach beyond BDMA technique is spelled out by recognizing the case of the base station communicating with the mobile stations ${ }^{[22]}$. In this communication, an orthogonal beam is assigned to every mobile station, and BDMA approach will fractionate that antenna beam on the report of the mobile stations' locations for granting multiple access to the mobile stations, which subsequently enlarges the magnitude of the system ${ }^{[23]}$.

There is no obligation to have a change in the wireless composition and setup, which had taken place in all mobile networks from $1 \mathrm{G}$ to $4 \mathrm{G}$. Alternatively, there could be only the addition of an application or improvement done at the fundamental network to please user requirements. To meet the demands of the user and answer the challenges that have been put forward in the $5 \mathrm{G}$ system, a radical alteration in the approach of designing the $5 \mathrm{G}$ wireless cellular architecture is needed ${ }^{[20]}$. A common saying of the researchers has been revealed in Ref. [24] that nearly all of the wireless users stay indoors for more or less than 80 percent of the time and 20 percent outdoors. In ongoing wireless cellular architecture, an outside base station presents in the middle of a cell and aids in connecting user inside or outside ${ }^{[19]}$. So for inside users to connect with the outside base station, the signals have to go through the walls which respectively expenses and lowers the spectral competence, data flow, and energy efficiency ${ }^{[25]}$ 
of wireless communications ${ }^{[20]}$.

To overpower this encounter, a new designing scheme is adopted for $5 \mathrm{G}$ architecture (see Fig. 1) to define outside and inside operations ${ }^{[23]}$. With this scheme, the infiltration casualty past walls will be faintly reduced. Massive MIMO technology ${ }^{[26]}$ will help to achieve this idea, in which geographically outlying ranges of antennas are spread out. Meantime existing MIMO systems are using two to four antennas; although the concept of massive MIMO systems has turned up with the idea of making use of the assets of the considerable array antenna in the appellation of massive capacity gains. Due to massively connected devices, several encounters come into view which will be answered by expanding capacity and by improving energy efficiency ${ }^{[25]}$, cost, and spectrum exhaustion along with providing superior scalability for managing the growing number of connected devices.

To provide a common connected platform for a range of applications and requirements for $5 \mathrm{G}$, some solutions are identified as follows ${ }^{[27]}$ :

- D2D communications: which refer to direct communication among devices allowing the local interchange of user plane traffic without going through a network infrastructure.

- Massive Machine Communications (MMCs): which structure the support of the IoTs with a different application ranges counting the automotive industry, public safety, and eHealth.

- Moving Networks (MNs): that improve and extend linking together potentially large populations of

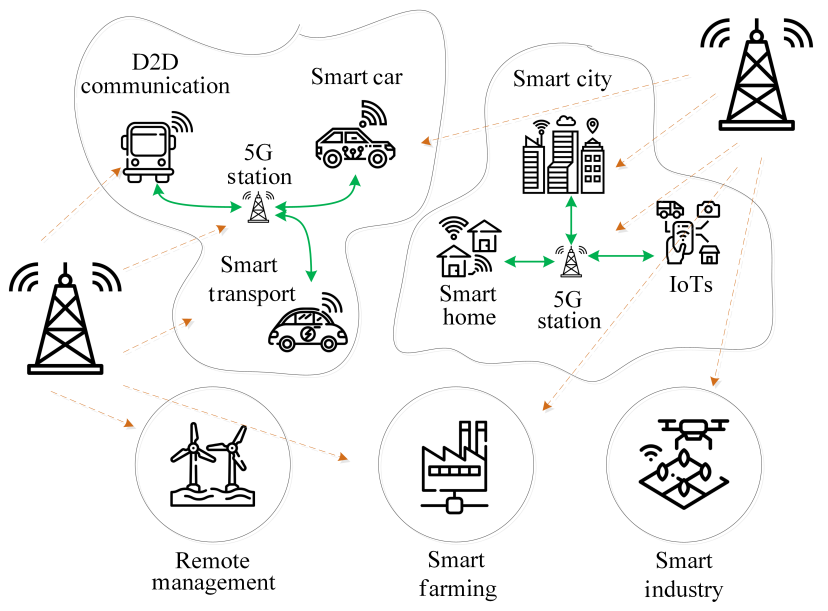

Fig. 1 A general 5G cellular network architecture. mutually moving communication devices.

- Ultra-Dense Networks (UDNs): which are the main driver whose goals are to increase capacity, upsurge energy efficiency of radio links, and empower superior exploitation of under-utilized spectrum.

- Ultra-Reliable Networks (URNs): that empower high availability.

\subsubsection{Cognitive network}

The community of networking research expects a new network technology called the cognitive network ${ }^{[28,29]}$. The word cognitive refers to "an entity that can accomplish some kinds of conscious intellectual activities, such as thinking, reasoning, learning, or remembering in order to make sense of the surroundings". Architectures of this approach encompass a cognitive process that can sense current situation, make choices and react consequently, and pilot the prospect. It is overall agreed that the ability to remember, to learn, and to think exists in cognitive networks $^{[30,31]}$.

The competences of a cognitive network can be highly distributed or extremely centralized depending on the engineering adjustments for each specific network. As a whole, distributed cognitive entities (agents) make the cognitive network where they are somehow "intelligent" in how they have specific cognitive facilities and are connected to the network ${ }^{[32]}$.

The Cognitive Radio (CR) techniques are the key permissive technologies of CR networks, which furnish the effectiveness to share the spectrum in strategic practice. CR also can switch its transmitter parameters to be used as a basis for the interaction and influence of its environment ${ }^{[33]}$. Formally, pair main features of cognitive radio can be discussed ${ }^{[34]}$ :

- Cognitive capability: The spectrum segments that are unexploited at a precise time or location can be discovered by way of real-time interaction with the radio milieu. Hence, the ideal spectrum can be identified, selected, and shared with other users, and utilized without tampering with the accredited user.

- Re-configurability: A CR can be set up to send and receive on a sort of frequencies. It uses different access technologies succored by its hardware design ${ }^{[35]}$. The ideal spectrum band and the extremely fitting operating 
constraints can be determinated and recomposed using the above ability.

The CR network does not have a license to operate in a desired band. Therefore, additional functionality is required for CR users to share the licensed spectrum band. Also, extra functionality is needed for CR users to share the permitted spectrum band. CR base stations are the CR network components that supply single-hop connection to CR users ${ }^{[36]}$.

CR users can access both the licensed and the unlicensed fragment of the spectrum allocated by primary users through wideband access technology. As a result, the operation types for CR networks can be classified as licensed band operation and unlicensed band operation.

- Licensed band operation: The primary network uses the licensed band. For this purpose, CR networks mainly focus on the identification of licensed users in this case. The channel aptitude revolves around the interference at neighboring licensed users. Besides, if primary users become visible in the spectrum band engaged by $\mathrm{CR}$ users, CR users should reverse that spectrum band and proceed to obtainable spectrum instantly.

- Unlicensed band operation: In the absence of primary users, CR users have the same privilege to access the spectrum. Henceforth, sophisticated spectrum sharing methods are required for CR users to take part in the unlicensed band ${ }^{[37]}$

Cognitive network cycle, as illustrated in Fig. 2, comprising recognizing spectrum white space, picking up the ideal frequency bands, and commanding spectrum access with other users and abandoning the frequency

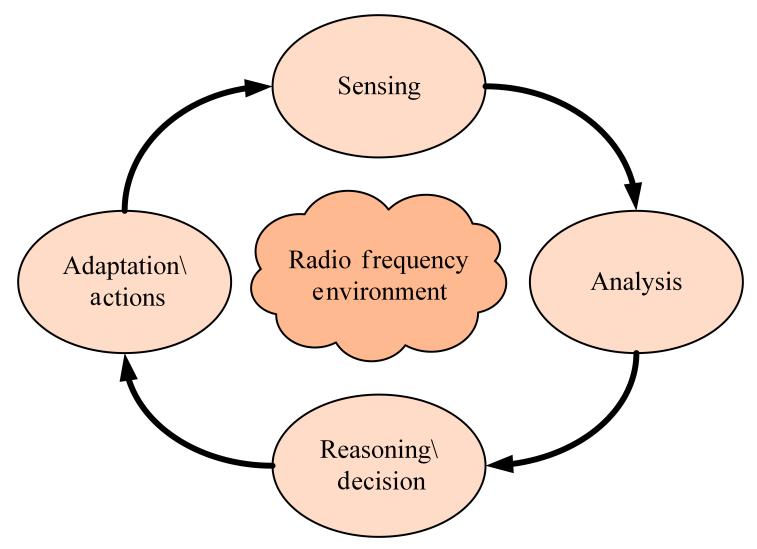

Fig. 2 Cognitive cycle. when a licensed user shows up. The aforementioned cognitive cycle is supported by the succeeding functions:

- Spectrum sensing and analysis;

- Spectrum management and handoff; and

- Spectrum allocation and sharing.

Secondary users can settle on the ideal frequency band and hop in the multiple bands using spectrum management and handoff basis of $\mathrm{CR}$ after recognizing the spectrum white space by sensing. They choose on the report of the time varying channel features to meet QoS requirements ${ }^{[38]}$. For instance, the secondary user that operates on the licensed band can direct his/her communication to other accessible frequencies when a licensed user retrieves his/her frequency band according to the spectrum capacity determined by the noise and interference levels, channel error rate, etc.

In dynamic spectrum access, an unlicensed user may share the spectrum band with licensed users, other unlicensed users, or both. For that reason, a good spectrum designation and sharing procedure are pivotal to achieve high spectrum adaptability. Since licensed users hold the spectrum privilege, when secondary users co-exist in a licensed band with licensed users, the fading level due to unlicensed spectrum usage should be bounded by a certain threshold. When multiple secondary users share a frequency band, their access should be harmonized to alleviate collisions and interference ${ }^{[39]}$.

\subsubsection{Virtual network}

Virtualization technology is to simulate a hardware device or unit to software resource. Virtualization is also to imitate all the functionalities of hardware and simulate them as a "virtual instance" with the ability to operate just like the hardware solution would.

"Network Virtualization (NV) abstracts networking connectivity and services that have traditionally been delivered via hardware into a logical virtual network, which in turn, is decoupled from and runs independently on top of a physical network in a hypervisor (virtual machine monitor)". Network virtualization ${ }^{[40-42]}$ is one of the most potential technologies which will take the Internet to another level. It has already been actively used in research and applied in distributed cloud computing environment ${ }^{[43]}$. $\mathrm{NV}$, by now, is seen as a tool to 
overcome the resistance of the current internet to fundamental changes.

Virtualization comes up with the prospect for flexible software design. Common networking services are supported by different network functions that are linked in a static state. Network Function Virtualization (NFV) empowers further dynamic blueprints to create and administrate network functions. Its key conviction is the Virtual Network Function (VNF) forwarding graph, which removes the complexity from the service chain provisioning by speedily and inexpensively creating, modifying, and removing service chains.

Matched to current practice, NFV introduces the following three major dissimilarities ${ }^{[44]}$ :

- Separation of software from hardware: This separation enables the software to evolve independent from the hardware and vice versa.

- Adaptable deployment of network functions: NFV can unconsciously set up network function software on a pool of hardware resources that may operate varied functions at diverse times in different data centers.

- Dynamic service provisioning: Network operators can scale the NFV competence in a robust way and on a grow-as-you-need assumption with fine granularity control constructed on the existing network conditions.

According to European Telecommunications Standards Institute (ETSI), the NFV architecture is levelheaded of three fundamental elements: Network Function Virtualization Infrastructure (NFVI), VNFs, and NFV MANO ${ }^{[44,45]}$. They are portrayed graphically in Fig. 3 and listed below as follows:

- NFVI: The NFVI is the merging of both hardware and software resources that structures the environment in which VNFs are installed. The physical resources comprise Commercial-Off-The-Shelf (COTS) computing hardware, storage, and network (both nodes and links) that affords processing, storage, and connectivity to VNFs. Virtual resources are notions of computing, storage, and network staff. The presumption is achieved by employing a virtualization layer (based on a hypervisor) which dissociates the virtual resources from the original physical resources. In a data center milieu, the computing and storage resources may be

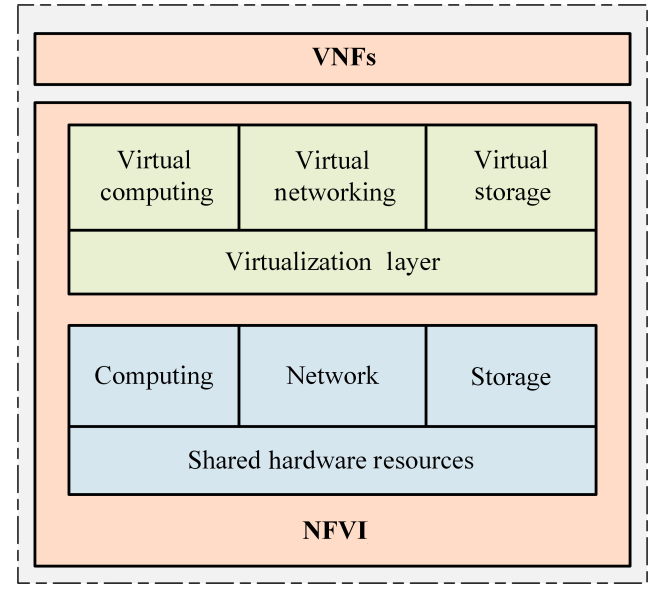

Fig. 3 NFV architectural framework.

characterized in terms of more than one Virtual Machine (VM), while virtual networks are made up of virtual links and nodes. A virtual node is a software portion with either hosting or routing functionality for illustration an operating system encapsulated in a VM. A virtual link is a logical interconnection of two virtual nodes. For them, it looks as a direct physical link with dynamically changing properties ${ }^{[46]}$.

- Virtual network functions and services: An Network Function (NF) is a functional block within a network infrastructure that has well-defined external interfaces and functional behavior ${ }^{[45]}$. Accordingly, a $\mathrm{VNF}$ is an implementation of an NF that is used on virtual resources, such as a VM. A single VNF may be composed of various internal modules, and hence, it could be deployed over innumerable VMs, in which case each VM hosts a single component of the $\mathrm{VNF}^{[44]}$. A service is a subscription delivered by a Telecommunications Service Provider (TSP) that is composed of one or more NFs. In the case of NFV, the NFs that make up the service are virtualized and used on virtual resources, such as a VM. However, from the users view, the services - whether based on functions running dedicated equipment or on VMs should have the same conduct. The number, type, and ordering of VNFs that make it up are governed by the service's functional and behavioral framing. Therefore, the behavior of the service is dependent on that of the constituent $\mathrm{VNF}^{[47]}$.

- NFV MANagement and Orchestration (NFV MANO): Following to the ETSI's MANO framework ${ }^{[48]}$, NFV MANO delivers the mandatory functionality 
for the provisioning of VNFs and the interconnected operations, such as the configuration of the VNFs and the infrastructure where these functions run on. It consists of the orchestration and lifecycle management of physical and/or software assets that upkeep the infrastructure virtualization and the lifecycle management of VNFs. It also takes into account the databases that are deployed to store the information and data models that outline both deployments and lifecycle properties of services, resources, and functions. NFV MANO focuses on all virtualization-specific management tasks necessary in the NFV framework. The framework illuminates interfaces that can be used for communications among the dissimilar components of the NFV MANO and the synchronization with traditional network super visioning systems, such as Operations Support System (OSS) and Business Support Systems (BSS), to authorize for management of both VNFs and functions running on legacy equipment ${ }^{[47]}$.

\subsubsection{Software defined networking}

The communications engineering has originated and established new technologies adjacently related to NFV which are cloud computing and SoftwareDefined Networking (SDN). Following to the National Institute of Standards and Technology (NIST) ${ }^{[49]}$, cloud computing is "a model for enabling ubiquitous, convenient, and on-demand network access to a shared pool of configurable computing resources (e.g., networks, servers, storage, applications, and services) that can be rapidly provisioned and released with minimal management effort or service provider interaction". In a cloud computing setting, the gray aspect of the service supplier is split into the infrastructure providers and service providers. Infrastructure providers administrate cloud platforms and lease resources corresponding to a usage-based pricing plan, whereas service providers rent resources from one or countless infrastructure suppliers to serve the end users ${ }^{[50]}$. We will discuss the cloud computing in the computing section. $\mathrm{SDN}^{[51]}$, at the current time, is charming the attention from both academia and industry as a chief architecture for the administration of massive scale complex networks, which may necessitate re-policing or re-configurations routinely. As shown in Fig. 4, SDN disengages the network control and forwarding functions. This authorizes network supervision to become right away programmable via an open interface (e.g., ForCES ${ }^{[52]}$, OpenFlow ${ }^{[53]}$, etc.) and the primary infrastructure to become elementary packet forwarding devices (the data plan) that can be programmed.

SDN has the aptitude to intelligible network administration and empowers innovation and progression $^{[54]}$. According to the Open Network Foundation (ONF) ${ }^{[55]}$, SDN focuses on the fact that the static architecture of conventional networks is unfitting for the dynamic computing and storage necessitates of today's data centers.

The SDN architecture is as follows ${ }^{[56]}$ :

- Programmable: SDN makes network control programmable since control is dissociated from forwarding functions. This programmability can be employed to automate network configuration in such a way that network proprietors can run "SDN apps" that helps to enhance certain services, such as VoIP, to ensure a high Quality-of-Experience (QoE) for phone calls.

- Agile: Splitting control from forwarding lets network supervisors dynamically alter network-wide traffic flow to meet changing demands. It frames the network to be more agile since logic is now implemented in software running on commodity hardware, which has shorter release cycles than device firmware.

- Centrally managed: Network brainpower is (logically) centralized in software-based SDN controllers that maintains a global view of the network, which appears to applications and policy engines as a single logical switch.

- Open standards-based and vendor-neutral: When implemented through open standards, SDN simplifies network design and operation because instructions are provided by SDN controllers in lieu of numerous definite devices and procedures.

\begin{tabular}{|c|c|c|}
\hline \multirow{2}{*}{$\begin{array}{r}\text { SDN } \\
\text { layers }\end{array}$} & Application layer & Business applications \\
\cline { 2 - 3 } & Control layer & Network services \\
\cline { 2 - 3 } & Infrastructure layer & Network infrastructure \\
\hline
\end{tabular}

Fig. 4 Logical layers in a software defined network. 


\subsection{Development trends of computing}

\subsubsection{Cloud computing}

Cloud computing is an emerging computation model that provides on-demand services and shared resources over the Internet. Cloud computing, based on large storage and computational devices, acts as a utility provider ${ }^{[57,58]}$. "A large-scale distributed computing paradigm that is driven by economies of scale, in which a pool of abstracted, virtualized, dynamically-scalable, managed computing power, storage, platforms, and services are delivered on demand to external customers over the Internet"[59].

There are minor fundamental points in this definition. First, cloud computing is a distributed computing paradigm. It is dissimilar from classic ones as follows: (1) it is tremendous scalable; (2) it can be encapsulated as an abstract entity that delivers different levels of services to customers outside the cloud; and (3) the services can be dynamically configured (via virtualization or other approaches) ${ }^{[60]}$.

The taxonomy of clouds (shown in Fig. 5) consists of different participants involved in the cloud along with the aspects and technologies that are incorporate to address their needs. The altered types of services "XaaS" are in the following ${ }^{[61,62]}$ :

- Software as a Service (SaaS): These are application software deployed on remote servers by providers as services to be accessed by consumers (end users) over the Internet. Examples of SaaS are E-mail services, social media, salesforce.com, etc.

- Platform as a Service (PaaS): These are cloud frameworks that are made available by services providers to be used for application development, testing, and deployment by consumers. Some examples of PaaS

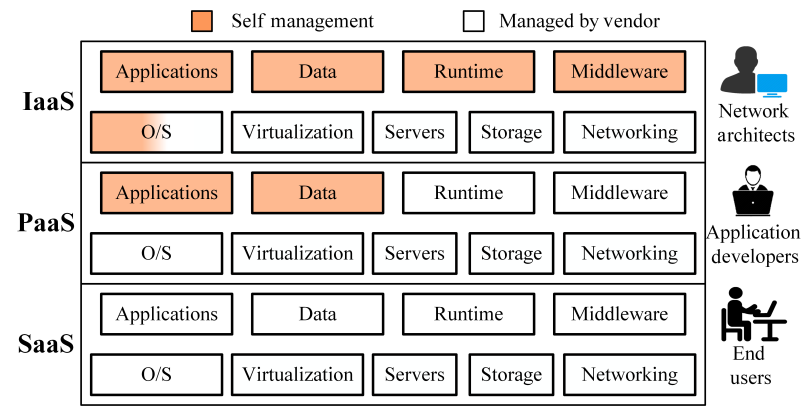

Fig. 5 Cloud computing taxonomy. are Google App Engine, Microsoft Azure, Maqetta, Appistry's CloudIQ, etc.

- Infrastructure as a Service (IaaS): This is ondemand computing infrastructure made available to consumers mostly in the form of virtualized servers, storage, and network. Some examples of IaaS are Alibaba Compute Cloud, iCloud, Microsoft Azure, etc.

On the other hand, the cloud can also be categorized, conditionally on the deployment models, as private, public, community, and hybrid ${ }^{[49]}$.

- Private cloud: The cloud is owned by a provider company, and the information is shared only within the organization. The justification of this type of cloud application is to fulfill its business applications.

- Public cloud: On the other hand, the public cloud is owned by a service provider and used by the public for their purposes.

- Community cloud: Community cloud is similar to the private cloud with some additional features to provide services to a group of organizations who have similar type of requirements.

- Hybrid cloud: Hybrid cloud is the extension of cloud computing with private, public, and community cloud computing techniques ${ }^{[63]}$. The private, public, and community clouds are integrated together to perform several tasks which are capable of handling the requirements of private, public, and community organizations.

\subsubsection{Edge computing}

Edge computing alludes to the qualifying technology empowering computation to be effectuated at the edge of the network, on downstream data on the assistance of cloud services and upstream data on the assistance of IoT services. For example, a smartphone is an edge between wearable devices and the cloud, a gateway in a smart manufacture is the edge between manufacture equipment and the cloud. The rational of edge computing is that computing should happen at the vicinity of data sources $^{[64]}$.

Why do we need edge computing? Firstly, putting all the computing tasks on the cloud has been showed to be an ineffective manner for data processing since the computing potential of the cloud overreach the competence of the things at the edge. Secondly, nearly 
all kind of electric devices will become part of IoT where the number of the things at the extremity of the network will grow to more than billions in a few years. This signifies most of data bring out by IoT will never be communicated to the cloud. Alternatively, it will be consumed at the edge of the network ${ }^{[64]}$.

Mobile Edge Computing (MEC) is an emerging technology in $5 \mathrm{G}$ era which allows the running of the cloud and IT services nearby to mobile users. It allows the availability of the cloud servers inside or adjacent to the base station. The end-to-end latency anticipated by the users' devices is accordingly made lower with the MEC platform ${ }^{[65]}$.

MEC enables an innovative network architecture where cloud computing capabilities and an IT service environment are converged with the cellular network for application delivery. Its benefits stem from the unique characteristics differentiating it from a typical cloud platform in the following aspects:

- Extreme proximity to the user (typically just one or two network hops away) which enables the significantly reduced end-to-end latency and the alleviated core network congestion.

- Radio network capability exposure including the access to network information and the integration with operator's network services to application developers.

These features allow the mobile network operators, vendors, as well as application service providers to improve existing services and boost new ones enabling significant complementary value added to their respective business models.

MEC, shown in Fig. 6, refers to the high performance and Telco-grade cloud platform adjacent to the Radio Access Network (RAN) allowing the computation to happen at the network edge. It works on both the downstream data from the cloud service host to the mobile terminal and the upstream data from the mobile terminal to the cloud host ${ }^{[66]}$.

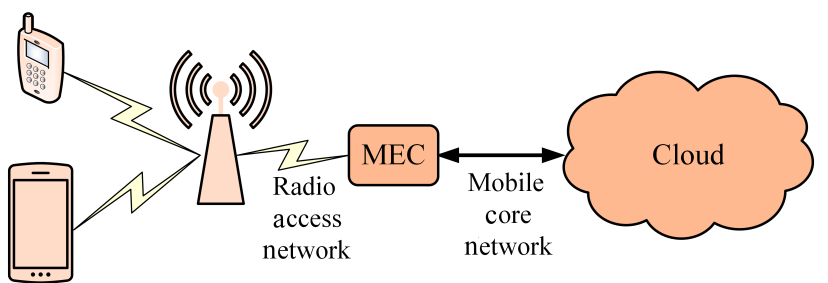

Fig. 6 MEC architecture.
The MEC platform is composed of the standard IT servers and the network devices inside or outside of the base station. The 3rd party applications are deployed and executed within the $\mathrm{VMs}^{[67]}$ interconnected by the network devices. It is also possible to build the MEC platform simply with the standard IT servers where the network device is implemented as the software entity, such as Open vSwitch (OVS) ${ }^{[68]}$.

The fundamental functionalities in the MEC platform include routing module, network capability exposure modular, and management modular. The routing modular is responsible for the packet forwarding among the MEC platform, the RAN, and the mobile core network, as well as within the MEC platform. The network capability exposure modular enables the authorized exposure of the Radio Network Information Service (RNIS) and Radio Resource Management (RRM). The management modular supports the Authentication, Authorization, and Accounting (AAA), and management of the 3rd applications in the MEC platform. In particular, it involves the orchestration for the application deployment and the authorization on the network capability exposure ${ }^{[66]}$.

\subsubsection{Fog computing}

Fog computing is a virtualized platform that assists the progress of the management of computing, network administration, and storage services among data centers and end devices as shown in Fig. 7, commonly, but not mainly located at the edge of the network. It powers cloud and edge resources along with its

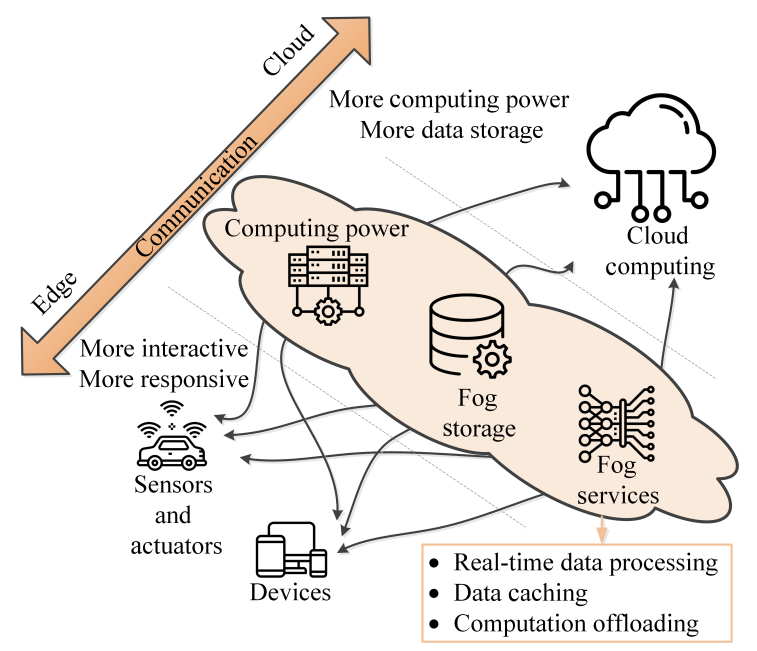

Fig. 7 Fog computing architecture. 
particular infrastructure ${ }^{[69]}$. Fog computing encompasses the elements of data-processing or analytics applications operating in the distributed cloud and edge appliance. As well it supports user movability, interface and resource assortment, and distributed data analytics to root for the requirements of a distributed application that it demands low latency ${ }^{[70]}$.

Why do we need fog computing? We need it to minimize latency and conserve network bandwidth, address security concerns at all level of the network, operate accurately with quick outcomes, collect and secure vast array of data, shift data to the ideal site for processing, lower expenses of using high computing power only when needed and less bandwidth, excelling analysis and insights of local data. In Fig. 8, fog systems largely use the sense process actuates and stream processing programming models. Sensors stream data to IoT networks, applications running on fog devices subscribe and process the information, and the acquired insights are translated into actions, which in turn, are sent to actuators ${ }^{[70]}$.

Fog systems dynamically explore and use APIs to frame convoluted functionalities. Components at the resource management layer maneuver information from the resource monitoring service to pathway the state of available cloud, fog, and network resources and precise the best candidates to process incoming tasks. With multitenant applications, the resource management components prioritize the tasks of the assorted participating users or programs ${ }^{[71,72]}$.

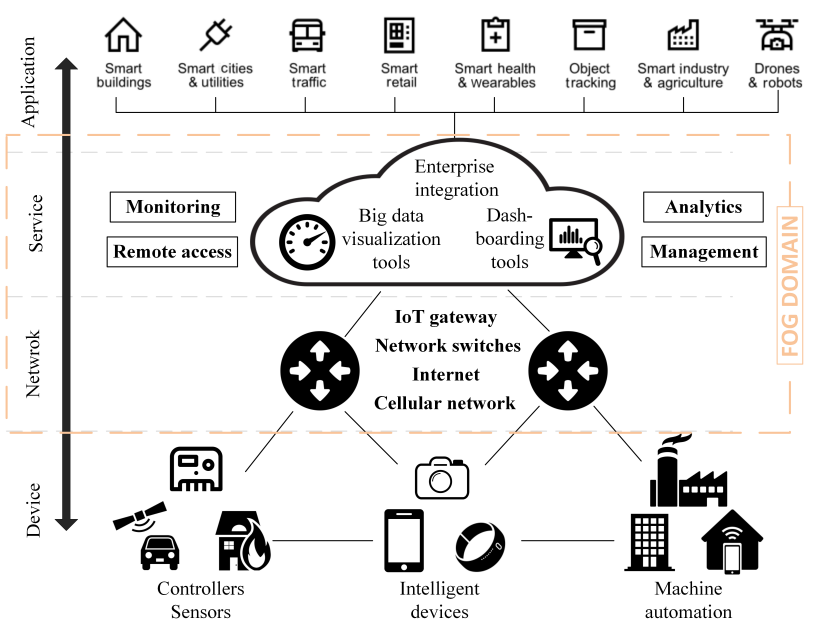

Fig. 8 Fog computing reference architecture.
Edge and cloud resources communicate using Machine-to-Machine (M2M) standards, such as Open Mobile Alliance Light Weight M2M (OMA LWM2M) and the Constrained Application Protocol (CoAP). SDN helps with the efficient management of heterogeneous fog networks ${ }^{[70]}$.

The application of fog computing can be viewed in various domains as follows:

- Healthcare and activity tracking: Fog computing could be suitable for healthcare, in which real-time processing and event response are decisive. One proposed system makes use of fog computing to observe, anticipate, and avoid collapse by stroke patients. Experiments concluded that this system had a slower response time and consumed less energy than cloudonly approaches. A suggested fog computing occupying smart healthcare system conducts to low latency, movability support, failure detection, and location and privacy perception ${ }^{[70]}$.

- Smart utility services: Fog computing can be used with smart utility services, whose concentration is ameliorating energy generation, distribution, and posting. In such a context, edge appliance can record more fine grained energy utilization details (for example, hourly, daily, and weekly, rather than monthly readings) to users' mobile devices than long-established smart utility services. These edge devices can also quantify the cost of power consumption during the whole day and convey advice about which energy source is most economical at any given time or when office appliances should be turned on to minimize utility use ${ }^{[70]}$.

- Augmented reality, cognitive systems, and gaming: Fog computing performs a major role in boosting reality applications, which are latency delicate. For example, the electroencephalogram (EEG) tractor beam augmented multiplayer, which is an online brain-computer interaction game, performs continuous real-time brain state classification on fog devices and then tunes classification models on cloud servers based on EEG readings that sensors assemble.

A wearable cognitive facilitation system that uses Microsoft HoloLens appliance aids bodies with reduced mental acuity performs numerous tasks, including telling 
them the places they have been and do not remember. In consideration of time sensitive engagements, the system streams video from the glass camera to the nearest fog for processing. The system reveals how using nearby fogs significantly drops end-to-end latency ${ }^{[70]}$.

\section{Convergence of caching in contemporary enabling technology and trend}

In this section, we have explored the cutting-edge technologies that been introduced or enhanced in terms of achieving convergence of CCC in IoT. The following listed technologies support the growth of the IoT and take into consideration its scalability, resource and data accessibility, storage capacity, computation power, and access control over a massive number of users.

\subsection{G network}

By developing the $5 \mathrm{G}$ network, the researchers expect it to provide the connecting users and things in IoT with high bandwidth, low delay, reliable communication, and less power consumption. The main focus of this technology is on the $5 \mathrm{G}$ cellular architecture, gigantic divers input and output technology, and D2D communication. Mobile and wireless networks have made remarkable development in the last few years, the revelation of $5 \mathrm{G}$ wireless communications lies in providing very high data rates, manifold increase in base station capacity, and significant upgrading in users' perceived QoS. SDN ${ }^{[73]}$ and VN are extensively deployed in $5 \mathrm{G}$ architecture design to overcome the upcoming challenges to support $\operatorname{IoT}^{[19]}$.

\subsection{Cloud computing}

Cloud computing is an information processing paradigm that supports a broad set of specific services and offers shared resources and significant computing power along with huge storage repositories at one platform over the Internet. The critical technology of cloud computing is "virtualization" that makes a single hardware system show as multiple virtual entities in terms of sharing computation capabilities and other resources. Besides, the cloud computing system is flexible, i.e., if the computing load required by an organization exceeds the computation power of a selected server, more resources from other servers can be assigned virtually in a transparent manner to ensure QoS to users. The integration of cloud computing and IoT opens wide doors in front of the innovative scenarios, especially the social and commercial applications ${ }^{[74]}$.

\subsection{Fog computing}

Fog computing is an additional structure added in the middle between the cloud and the end-users. It supports the cloud by reducing the computation and communication operations done on its side, and at the same time, make the resources being managed locally near to users. It provides computing, network operations, and storage services between cloud and devices like mobile phones, wearables devices, sensors, and cars $^{[75]}$. Besides, it offers data-processing or analytics applications for some cloud and edge appliance. Fog computing has already been used in many application domains, such as data collection, online gaming, streaming, healthcare, and smart utility services, to minimize latency substantially. It shows advantages in saving the network bandwidth, addressing security issues at all the levels of the network, and reducing the computing overload transferred to the cloud by operating accurately with quick outcomes ${ }^{[76,77]}$.

\subsection{Edge computing}

The main idea behind edge computing is keeping network services, computing power, and data storage on the edge of the network near to the end-users. The enormous growth of IoT devices gives no choice for achieving less delay except that most of the data can be processed at the edge of the network instead of transmitting to the cloud. Existing cellular network architecture performs control and user/data plane at the base station locally. However, handling the massive traffic from the significant number of devices in IoT was not taken under consideration. To resolve such issues, SDN can be set up at edge level to manage network traffic and resources with more flexibility and scalability support $^{[78]}$. In IoT, tha edge computing can be anywhere on the network edge; for example, the smartphone can be an edge between wearable devices and cloud. Moreover, the fog server is also an edge between cellular networks and the cloud. 


\section{Convergence inside smart physical devices of IoT}

\subsection{Sensor}

A sensor is an appliance that senses and reacts to some specific facts from the real environment. The explicit input could be motion, heat, moisture, light, pressure, etc. The output is mainly a signal that is converted to human-readable display at the sensor placement or diffused electronically over a network for displaying or additional processing and analysis ${ }^{[79]}$.

A smart sensor (computing and communication converged sensor) is a device that receipts data from the physical milieu and uses built-in compute properties to execute predefined functions upon detection of particular input and then processes the data before passing it on. The smart sensor is crucial and essential element in the IoTs. It is made of a sensor, a microprocessor, and a communication unit as illustrated in Fig. 9. The computing is required to be an indispensable part of the physical design. The sensor that just spreads its output onward for remote processing is not reflected as a smart sensor $^{[80,81]}$.

Wind farm is the best example to understand the difference between sensors and smart sensors. The wind turbine is a very sophisticated piece of equipment, depending on the weather. The turbines have to be changed in real-time in order to optimize the electricity output. Most of wind firms are located in remote areas where stable networks and cloud connectivity sometimes are not available. Smart sensors (edge intelligence) allow the data to be processed locally in actual time without having faith on continuous wide area network availability.

\subsection{Device}

An IoT device is any nonstandard computing device that connects wirelessly to a network and has the capability

\begin{tabular}{|l|c|}
\hline Data transmission & Communication unit \\
\hline Data processing & Microprocessor \\
\hline Data collection & Sensor chip \\
\hline
\end{tabular}

Fig. 9 IoT smart sensing device. to transmit or receive data. These things might have powerful CPUs, such as thermostats, light bulbs, home appliances (washers, dryers, refrigerators, etc.), and cars, or it might be a smart phone. The notion beyond the ubiquitous Internet is all of these appliances working in concert for people in businesses, industry, or at home. An example of a smart home enabled by IoT smart devices: The user arrives home, then, his phone communicates with the house to light the rooms. Due to the sensing of his proximity and his pacemaker data that shows it is been a stressful day when he walks through his room, the light will be adjusted to lower intensity and changed to his chosen color for relaxing ${ }^{[81]}$.

IoT devices are parts of a scenario in which every device talks to all other interconnected devices in a milieu to automate home and industry and communicate more and more serviceable data to users, businesses, and other interested parties ${ }^{[82]}$.

The most common challenges between sensors and devices are in the following: (1) Connectivity: Variety of wired and wireless connectivity standards are required to enable different application needs; (2) Reducing energy consumption: Many IoT smart sensors and devices need to run for years using batteries; (3) Security is vital: As the aim of IoT is to connect more appliances, it furnishes more decentralized opening points for malware, more layers of software, integration middleware, APIs, machine-to-machine communication, etc., create more complexity and new security risks.

\subsection{Router and gateway}

A gateway- “an earlier term for router", is a network node connecting two networks that use different base protocols. In enterprises, the gateway is the data processing machine that routes the traffic from offices to the outside network. In homes, the gateway is the Internet Service Provider (ISP) that links users to the Internet. Gateways can take several forms where it can be entirely implemented in software, entirely in hardware, or as a combination of both, and can perform a variety of tasks.

The gateway device relating things in IoT to the cloud should have additional functionality to do a little processing before transferring the data to the 
Internet and eventually to the cloud. Smart gateway has to accomplish various aspects of essential IoT, like, collecting, preprocessing, cleaning, filtering, and reforming the data into more beneficial form. Only the needed data will be transferred to the cloud. The gateway also has full control and monitoring of activities on IoT objects and sensors'. It observances the energy consumption of power nodes, ensures the security of data and the privacy of users, and performs general monitoring and management ${ }^{[83]}$. However, the management of M2M devices and endpoints also are powerfully done using smart gateways ${ }^{[84]}$.

\section{Convergence of computing, communication, and caching in IoT}

IoT is a network of physical appliances, cars, houses, and all other electronic devices, in addition to, computer programs. Mostly, IoT is wanted to offer advanced relatedness of electronic appliances, systems, and services that go beyond M2M and D2D communications and enclose an array of protocols, domains, and applications. In general, IoT requires sufficient support for ubiquitous communications and access to services and information. IoT empowers individuals and objects to be connected; anyone can connect anytime and anywhere with anything, and vice versa, using any available network, any service, or application. For that reason, IoT guarantees open access and conduct of the physical objects of the environment over the Internet.

We have explored some recent efforts in communication, computing, and caching technologies to answer the gap of convergence in future IoT. First, we can summarize a scenario of IoT in collecting data; after collecting massive information from physical world coming from diverse locations with the help of numerous existing and promising technologies (sensors, camera, microphone, etc.), the massive data needed to be aggregated without delay, indexed, stored, and processed more effectively, and then the data autonomously flow between other devices or to cloud. The idea of edge and fog computing is to distribute data to move it closer to the end user to eliminate latency, reduce high traffic, and support data streaming. In these two paradigms, the data is processed and stored close to users near to source which will lead to better end-users security. It is also valuable when service is provisioned from the data coming from the same location. In this approach, data process and service execute locally. This will lead to real time big data and real time analytics.

Cloud computing technology has faith in sharing resources, which is the main requirement for IoT platform. Cloud computing is not only about sharing the resources but also maximizing the resources. It is also location independent; the users access the cloud services from any location and with any devices through the Internet connection. When we talk about the IoT platform, then it should also be accessed from anywhere and anytime. The virtualization of physical devices is another important characteristic, which allows users to share the devices easily. The cloud computing paradigm enables sharing distributed resources to multiple users. In addition, the cloud offers elasticity and scalable of resources and application. The services and resources are easily accessible and available. For that reason, the convergence of cloud computing and IoT paradigm can furnish extensive opportunities for both technologies.

Rather than humans, devices also will communicate with each other independently without any centralized control and collaborate to collect, share, and forward information in a robust manner. The strength of gathering pertinent information in real-time is a key to worth IoT to maximum advantage, which will simplify the creation of an intelligent milieu. The quality of the gathered information depends on how intelligent and pertinent devices are. In addition, these communicating devices will operate with different networking standards, which may experience intermittent connectivity with each other, and many of them will be resource reserved. These physical attributes and existing characteristics open up numerous communications defy that customary routing protocols cannot puzzle out. The communication technologies are the backbone of future IoT. We explored some recent efforts in communication like $5 \mathrm{G}$, cognitive, virtual, and SDN networks, to mention the necessity of robust technologies to lead IoT in all layers as presented in Fig. 10. 


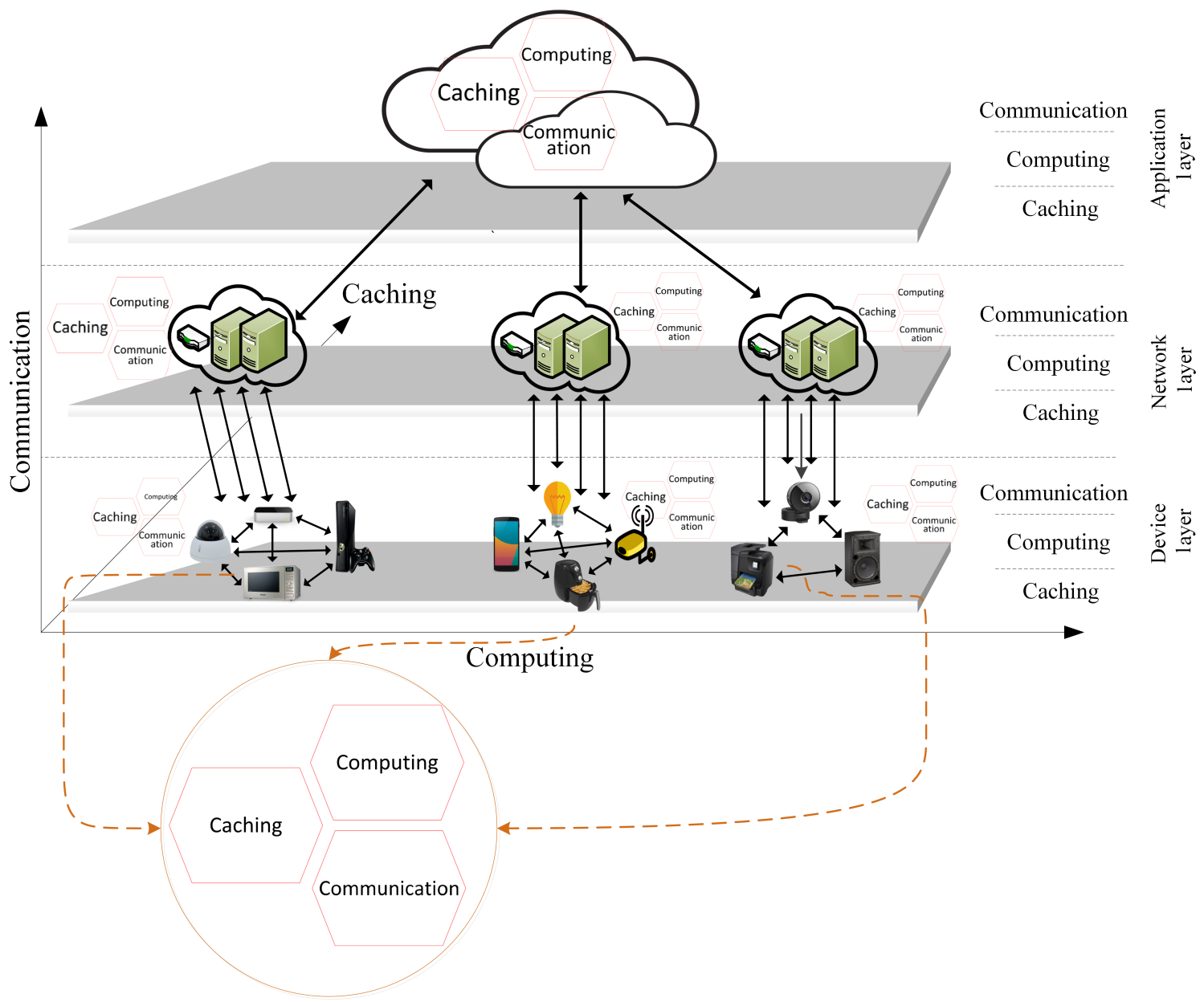

Fig. 10 Three dimensions (computing, communication, and caching) of IoTs.

\section{Future research direction and open problem}

The following, future research directions and open problems concerning the convergence of $\mathrm{CCC}$, are observed.

(1) Fog computing focuses processing efforts at the local area network end of the chain. Data are gathered, processed, and stored inner the network by manner of an IoT gateway or fog node. Information is diffused to this gateway from numerous sources in the network where it is processed and pertinent data, as well as any extra instructors, are transmitted back out to the mandatory devices while edge computing takes localized processing a bit farther pushing these struggles adjacent to the data source(s). Alternatively of doing the bulk of processing in a centralized server, each electronic appliance on the network would play its own role in processing the information. Fog and edge computing are promising technologies that will fully support convergence. Exploring more convergence of CCC in various technologies will lead to better understanding of converging technologies.

(2) Investigating data stream in convergence: Robust IoT may require gathering data continuously to determine certain readings to allow the operators to realize when they need to adjust the settings of a device and to control things in real-time. Data reduction is the process of underrating the volume of data that needs to be stored in data storage sites. Data reduction can increase storage efficiency and lower costs. A deep investigation of dataflow can lead to a solution of reducing data amount transfer between IoT devices and the backbone network and minimize the allocation of bandwidth.

(3) With unlimited deployment of sensors, the IoT 
will compose billions of devices that can detect, interact, compute, and potentially do actions. Devices with sensors to capture such information have existed for decades; the information is the sensed data related to temperature, orientation, motion, vibration, acceleration, humidity, chemical changes in the air, and so on, depending on the type of sensors. A virtualized SeNsor as a Service (SNaaS) may be pictured; in which users have the right of access and command their virtual private devices, and sequence of dissimilar sensors can be used for the design of smart services.

(4) Considering the role of convergence in sensors and actuate devices with the characteristics of different approaches, both can be used for a variety of applications. Some of these applications are naturaldisaster prophecy, water-scarcity monitoring, smarthomes design, medical applications, agricultural applications, intelligent transport system design, smartcities design, and smart metering and monitoring. Nevertheless, common users are daunted by the huge investments and high maintenance costs to utilize sensors. Shared sensors can be the solution, and applications, like selective sensing for sensors and service on-demand for actuating devices, can be investigated.

(5) Recent developments in technology have enabled the devices to be equipped with energy-efficient and cost-effective wireless modules that allow the devices to transmit data wirelessly in real-time. Examining the design of low power devices and realistic energy consumption models of different elements of IoT can lead to a better smart world.

\section{Conclusion}

The rapid growth of smart devices and the advent of many new applications have made computing task elephantine for devices, and the traffic volume has been increasing exponentially. The classic centralized network architecture cannot handle such user demands due to the heavy burden on the backhaul links and long latency. Therefore, new architectures, which converge network functions and computing tasks to domesticate the flashing growth of IT sector, have been introduced. The convergence can be viewed in three layers: cloud, pipe (communication channel), and the device in the edge. Fog computing needs 5G, SDN, and virtual networks, and edge devices need edge computing and cognitive network for better service.

In this paper, we have surveyed the convergence of $\mathrm{CCC}$ and the need for convergence. We have discussed recent efforts of communication in $5 \mathrm{G}$, cognitive and virtual networks, and computing in edge, fog, cloud technologies. We have also discussed the importance of convergence, and how we can lead IoT to be fully technologically converged with the help of promising communication, computing, and caching technologies. This paper also focuses on two key enablers of IoT elements, which are sensors and actuate devices, and coping those with communication, computing, and caching. Consequently, we have listed some future research directions in the convergence of CCC.

\section{References}

[1] A. Al-Fuqaha, M. Guizani, M. Mohammadi, M. Aledhari, and M. Ayyash, Internet of things: A survey on enabling technologies, protocols, and applications, IEEE Communications Surveys Tutorials, vol. 17, no. 4, pp. 2347 2376, 2015.

[2] M. Finnegan, Boeing 787s to create half a terabyte of data per flight, says virgin Atlantic, Computerworld $U K$, vol. 6, pp. 1-2, 2013.

[3] M. van Rijmenam, Self-driving cars will create 2 petabytes of data, what are the big data opportunities for the car industry? https:// datafloq.com/read/self-driving-carscreate-2-petabytes-data-annually/172, 2017.

[4] Data never sleeps 7.0, https://www.domo.com/learn/datanever-sleeps-7, 2019.

[5] T. E. of Encyclopaedia Britannica, Central processing unit | Definition \& Function | Britannica, https://global. britannica.com/technology/central-processing-unit, 2020.

[6] H. Hassan and M. Anis, Chapter 1-FPGA overview: Architecture and CAD, in Low-Power Design of Nanometer FPGAs, H. Hassan and M. Anis, eds. Boston, MA, USA: Morgan Kaufmann, 2010, pp. 1-29.

[7] A. Dunkels, B. Gronvall, and T. Voigt, Contiki-A lightweight and flexible operating system for tiny networked sensors, in Proc. of the 29th Annual IEEE International Conference on Local Computer Networks, Tampa, FL, USA, 2004, pp. 455-462.

[8] P. Levis, S. Madden, J. Polastre, R. Szewczyk, K. Whitehouse, A. Woo, D. Gay, J. Hill, M. Welsh, 
E. Brewer, et al., TinyOS: An Operating System for Sensor Networks. Berlin, Germany: Springer, 2005, pp. 115-148.

[9] Q. Cao, T. Abdelzaher, J. Stankovic, and T. He, The liteos operating system: Towards unix-like abstractions for wireless sensor networks, in Proc. of 2008 International Conference on Information Processing in Sensor Networks, St. Louis, MO, USA, 2008, pp. 233-244.

[10] E. Baccelli, O. Hahm, M. Günes, M. Wählisch, and T. C. Schmidt, RIOT OS: Towards an OS for the Internet of Things, in Proc. of 2013 IEEE Conference on Computer Communications Workshops , Turin, Italy, 2013, pp. 79-80.

[11] M. Ma, P. Wang, and C. Chu, Data management for internet of things: Challenges, approaches and opportunities, in Proc. of 2013 IEEE International Conference on Green Computing and Communications and IEEE Internet of Things and IEEE Cyber, Physical and Social Computing, Beijing, China, 2013, pp. 1144-1151.

[12] D. Farber and P. Baran, The convergence of computing and telecommunications systems, Science, vol. 195, no. 4283, pp. 1166-1170, 1977.

[13] D. G. Messerschmitt, The convergence of telecommunications and computing: What are the implications today? Proceedings of the IEEE, vol. 84, no. 8, pp. 1167-1186, 1996.

[14] M. Decina and V. Trecordi, Convergence of telecommunications and computing to networking models for integrated services and applications, Proceedings of the IEEE, vol. 85, no. 12, pp. 1887-1914, 1997.

[15] K. Kim and P. R. Kumar, Cyber-physical systems: A perspective at the centennial, Proceedings of the IEEE, vol. 100, pp. 1287-1308, 2012.

[16] P. Fan, Coping with the big data: Convergence of communications, computing and storage, China Communications, vol. 13, no. 9, pp. 203-207, 2016.

[17] I. Huang, R. Guo, H. Xie, and Z. Wu, The convergence of information and communication technologies gains momentum, The Global Information Technology Report, pp. 35-45, 2012.

[18] X. Foukas, G. Patounas, A. Elmokashfi, and M. K. Marina, Network slicing in 5G: Survey and challenges, IEEE Communications Magazine, vol. 55, no. 5, pp. 94-100, 2017.

[19] A. Gupta and R. K. Jha, A survey of 5G network: Architecture and emerging technologies, IEEE Access, vol. 3, pp. 1206-1232, 2015.

[20] M. Agiwal, A. Roy, and N. Saxena, Next generation $5 \mathrm{~g}$ wireless networks: A comprehensive survey, IEEE Communications Surveys Tutorials, vol. 18, no. 3, pp. 16171655, 2016.
[21] F. Schaich, T. Wild, and Y. Chen, Waveform contenders for $5 \mathrm{G}$-suitability for short packet and low latency transmissions, in Proc. of 2014 IEEE 79th Vehicular Technology Conference, Seoul, South Korea, 2014, pp. 1-5.

[22] J. G. Andrews, S. Buzzi, W. Choi, S. V. Hanly, A. Lozano, A. C. K. Soong, and J. C. Zhang, What will 5G be? IEEE Journal on Selected Areas in Communications, vol. 32 , no. 6, pp. 1065-1082, 2014.

[23] C. Wang, F. Haider, X. Gao, X. You, Y. Yang, D. Yuan, H. M. Aggoune, H. Haas, S. Fletcher, and E. Hepsaydir, Cellular architecture and key technologies for 5G wireless communication networks, IEEE Communications Magazine, vol. 52, no. 2, pp. 122-130, 2014.

[24] V. Chandrasekhar, J. G. Andrews, and A. Gatherer, Femtocell networks: A survey, IEEE Communications Magazine, vol. 46, no. 9, pp. 59-67, 2008.

[25] S. Buzzi, C.-L. I, T. E. Klein, H. V. Poor, C. Yang, and A. Zappone, A survey of energy-efficient techniques for $5 \mathrm{G}$ networks and challenges ahead, IEEE Journal on Selected Areas in Communications, vol. 34, no. 4, pp. 697-709, 2016.

[26] F. Rusek, D. Persson, B. K. Lau, E. G. Larsson, T. L. Marzetta, O. Edfors, and F. Tufvesson, Scaling up MIMO: Opportunities and challenges with very large arrays, IEEE Signal Processing Magazine, vol. 30, no. 1, pp. 40-60, 2013.

[27] A. Osseiran, F. Boccardi, V. Braun, K. Kusume, P. Marsch, M. Maternia, O. Queseth, M. Schellmann, H. Schotten, H. Taoka, et al., Scenarios for $5 \mathrm{G}$ mobile and wireless communications: The vision of the metis project, IEEE Communications Magazine, vol. 52, no. 5, pp. 26-35, 2014.

[28] D. D. Clark, C. Partridge, J. C. Ramming, and J. T. Wroclawski, A knowledge plane for the Internet, in Proceedings of the 2003 Conference on Applications, Technologies, Architectures, and Protocols for Computer Communications, New York, NY, USA, 2003, pp. 3-10.

[29] R. W. Thomas, D. H. Friend, L. A. DaSilva, and A. B. MacKenzie, Cognitive Networks. Dordrecht, the Netherlands: Springer, 2007, pp. 17-41.

[30] Q. Mahmoud, Cognitive Networks: Towards Self-Aware Networks. Chichester, England: Wiley, 2007.

[31] M. Youssef, M. Ibrahim, M. Abdelatif, L. Chen, and A. V. Vasilakos, Routing metrics of cognitive radio networks: A survey, IEEE Communications Surveys Tutorials, vol. 16, no. 1, pp. 92-109, 2014.

[32] C. Fortuna and M. Mohorcic, Trends in the development of communication networks: Cognitive networks, Computer Networks, vol. 53, no. 9, pp. 1354-1376, 2009.

[33] Docket no. 03-322 notice of proposed rule making and 
order, http://www.fcc.gov, 2003.

[34] S. Haykin, Cognitive radio: Brain-empowered wireless communications, IEEE Journal on Selected Areas in Communications, vol. 23, no. 2, pp. 201-220, 2005.

[35] F. K. Jondral, Software-defined radio-basics and evolution to cognitive radio, EURASIP Journal on Wireless Communications and Networking, vol. 2005, no. 3, p. 652784, 2005.

[36] O. Ileri, D. Samardzija, T. Sizer, and N. B. Mandayam, Demand responsive pricing and competitive spectrum allocation via a spectrum server, in Proc. of the First IEEE International Symposium on New Frontiers in Dynamic Spectrum Access Networks, Baltimore, MD, USA, 2005, pp 194-202.

[37] I. F. Akyildiz, W. Lee, M. C. Vuran, and S. Mohanty, A survey on spectrum management in cognitive radio networks, IEEE Communications Magazine, vol. 46, no. 4, pp. 40-48, 2008.

[38] I. F. Akyildiz, W.-Y. Lee, M. C. Vuran, and S. Mohanty, Next generation/dynamic spectrum access/cognitive radio wireless networks: A survey, Computer Networks, vol. 50, no. 13 , pp. 2127-2159, 2006.

[39] B. Wang and K. J. R. Liu, Advances in cognitive radio networks: A survey, IEEE Journal of Selected Topics in Signal Processing, vol. 5, no. 1, pp. 5-23, 2011.

[40] N. M. M. K. Chowdhury and R. Boutaba, Network virtualization: State of the art and research challenges, IEEE Communications Magazine, vol. 47, no. 7, pp. 20-26, 2009.

[41] A. Fischer, J. F. Botero, M. T. Beck, H. de Meer, and $\mathrm{X}$. Hesselbach, Virtual network embedding: A survey, IEEE Communications Surveys Tutorials, vol. 15, no. 4, pp. 18881906, 2013.

[42] K. Tutschku, T. Zinner, A. Nakao, and P. Tran-Gia, Network virtualization: Implementation steps towards the future internet, Electronic Communications of the EASST, vol. 17, pp. 1-14, 2009.

[43] P. T. Endo, A. V. de Almeida Palhares, N. N. Pereira, G. E. Goncalves, D. Sadok, J. Kelner, B. Melander, and J. Mangs, Resource allocation for distributed cloud: Concepts and research challenges, IEEE Network, vol. 25, no. 4, pp. 4246, 2011.

[44] G. ETSI, Network Functions Virtualisation (NFV): Architectural framework, ETSI Group Specifications NFV, vol. 2, no. 2, pp. 1-21, 2013.

[45] N. ETSI, Network Functions Virtualisation (NFV): Terminology for main concepts in NFV, Group Specification, vol. 3, pp. 1-10, 2014.

[46] R. Mijumbi, J. Serrat, J. Gorricho, N. Bouten, F. De Turck, and R. Boutaba, Network function virtualization: State- of-the-art and research challenges, IEEE Communications Surveys Tutorials, vol. 18, no. 1, pp. 236-262, 2016.

[47] R. Mijumbi, J. Serrat, J. Gorricho, N. Bouten, F. de Turck, and R. Boutaba, Network function virtualization: Stateof-the-art and research challenges, IEEE Communications Surveys Tutorials, vol. 18, no. 1, pp. 236-262, 2016.

[48] M. Ersue, RTSI NFV management and orchestrationAn overview, in Proc. of 88th IETF meeting, Vancouver, Canada, 2013, pp. 1-14.

[49] P. M. Mell and T. Grance, SP 800-145. The NIST definition of cloud computing. Gaithersburg, MD, USA: National Institute of Standards \& Technology, 2011.

[50] Q. Zhang, L. Cheng, and R. Boutaba, Cloud computing: State-of-the-art and research challenges, Journal of Internet Services and Applications, vol. 1, no. 1, pp. 7-18, 2010.

[51] F. Hu, Q. Hao, and K. Bao, A survey on software-defined network and openflow: From concept to implementation, IEEE Communications Surveys Tutorials, vol. 16, no. 4, pp. 2181-2206, 2014.

[52] A. Doria, J. H. Salim, R. Haas, H. M. Khosravi, W. Wang, L. Dong, R. Gopal, and J. M. Halpern, Forwarding and control element separation (ForCES) protocol specification, Heise Zeitschriften Verlag, vol. 5810, pp. 1-124, 2010.

[53] A. Lara, A. Kolasani, and B. Ramamurthy, Network innovation using openflow: A survey, IEEE Communications Surveys Tutorials, vol. 16, no. 1, pp. 493-512, 2014.

[54] B. A. A. Nunes, M. Mendonca, X. Nguyen, K. Obraczka, and T. Turletti, A survey of software-defined networking: Past, present, and future of programmable networks, IEEE Communications Surveys Tutorials, vol. 16, no. 3, pp. 1617 1634, 2014.

[55] ONF, Open networking foundation is an operator led consortium leveraging SDN, NFV and Cloud technologies to transform operator networks and business models, https://www.opennetworking.org/, 2017.

[56] M. Jarschel, T. Zinner, T. Hossfeld, P. Tran-Gia, and W. Kellerer, Interfaces, attributes, and use cases: A compass for SDN, IEEE Communications Magazine, vol. 52, no. 6, pp. 210-217, 2014.

[57] L. M. Vaquero, L. Rodero-Merino, J. Caceres, and M. Lindner, A break in the clouds: Towards a cloud definition, SIGCOMM Comput. Commun. Rev., vol. 39, no. 1, pp. 50-55, 2009.

[58] P. V. Krishna, S. Misra, D. Joshi, and M. S. Obaidat, Learning automata-based sentiment analysis for recommender system on cloud, in Proc. of 2013 International Conference on Computer, Information and Telecommunication Systems, Athens, Greece, 2013, pp. $1-5$. 
[59] S. Bera, S. Misra, and J. J. P. C. Rodrigues, Cloud computing applications for smart grid: A survey, IEEE Transactions on Parallel and Distributed Systems, vol. 26 no. 5, pp. 1477-1494, 2015.

[60] I. Foster, Y. Zhao, I. Raicu, and S. Lu, Cloud computing and grid computing 360-degree compared, in Proc. of 2008 Grid Computing Environments Workshop, Austin, TX, USA, 2008, pp. 1-10.

[61] B. P. Rimal, E. Choi, and I. Lumb, A taxonomy and survey of cloud computing systems, in Proc. of 2009 Fifth International Joint Conference on INC, IMS and IDC, Seoul, South Korea, 2009, pp. 44-51.

[62] R. K. Lomotey and R. Deters, Architectural designs from mobile cloud computing to ubiquitous cloud computingsurvey, in Proc. of 2014 IEEE World Congress on Services, Anchorage, AK, USA, 2014, pp. 418-425.

[63] F. Luo, Z. Y. Dong, Y. Chen, Y. Xu, K. Meng, and K. P. Wong, Hybrid cloud computing platform: The next generation it backbone for smart grid, in Proc. of 2012 IEEE Power and Energy Society General Meeting, San Diego, CA, USA, 2012, pp. 1-7.

[64] W. Shi, J. Cao, Q. Zhang, Y. Li, and L. Xu, Edge computing: Vision and challenges, IEEE Internet of Things Journal, vol. 3, no. 5, pp. 637-646, 2016.

[65] M. T. Beck, S. Feld, C. Linnhoff-Popien, and U. Pützschler, Mobile edge computing, Informatik-Spektrum, vol. 39, no. 2, pp. 108-114, 2016.

[66] Y. Yu, Mobile edge computing towards 5G: Vision, recent progress, and open challenges, China Communications, vol. 13, no. 2, pp. 89-99, 2016.

[67] R. Buyya, J. Broberg, and A. Goscinski, Cloud Computing: Principles and Paradigms. Chichester, UK: John Wiley \& Sons, 2011.

[68] B. Pfaff, J. Pettit, T. Koponen, E. Jackson, A. Zhou, J. Rajahalme, J. Gross, A. Wang, J. Stringer, P. Shelar, et al., The design and implementation of open vSwitch, in Proc. of 12th USENIX Symposium on Networked Systems Design and Implementation, Oakland, CA, USA, 2015, pp. 117-130.

[69] F. Bonomi, R. Milito, J. Zhu, and S. Addepalli, Fog computing and its role in the internet of things, in Proceedings of the First Edition of the MCC Workshop on Mobile Cloud Computing, New York, NY, USA, 2012, pp. 13-16.

[70] A. V. Dastjerdi and R. Buyya, Fog computing: Helping the internet of things realize its potential, Computer, vol. 49, no. 8, pp. 112-116, 2016.
[71] P. Hu, H. Ning, T. Qiu, Y. Zhang, and X. Luo, Fog computing-based face identification and resolution scheme in internet of things, IEEE Transactions on Industrial Informatics, vol. 13, no. 4, pp. 1910-1920, 2017.

[72] P. Hu, H. Ning, T. Qiu, H. Song, Y. Wang, and $\mathrm{X}$. Yao, Security and privacy preservation scheme of face identification and resolution framework using fog computing in internet of things, IEEE Internet of Things Journal, vol. 4, no. 5, pp. 1143-1155, 2017.

[73] H. Farhady, H. Lee, and A. Nakao, Software-defined networking: A survey, Computer Networks, vol. 81, pp. 79-95, 2015.

[74] A. Botta, W. de Donato, V. Persico, and A. Pescapé, Integration of cloud computing and internet of things: A survey, Future Generation Computer Systems, vol. 56, pp. 684-700, 2016.

[75] T. H. Luan, L. Gao, Z. Li, Y. Xiang, and L. Sun, Fog computing: Focusing on mobile users at the edge, Computer Science, vol. abs/1502.01815, 2015.

[76] S. Yi, C. Li, and Q. Li, A survey of fog computing: Concepts, applications and issues, in Proceedings of the 2015 Workshop on Mobile Big Data, New York, NY, USA, 2015, pp. 37-42.

[77] P. Hu, S. Dhelim, H. Ning, and T. Qiu, Survey on fog computing: Architecture, key technologies, applications and open issues, Journal of Network and Computer Applications, vol. 98, pp. 27-42, 2017.

[78] S. Wang, X. Zhang, Y. Zhang, L. Wang, J. Yang, and W. Wang, A survey on mobile edge networks: Convergence of computing, caching and communications, IEEE Access, vol. 5, pp. 6757-6779, 2017.

[79] M. Rouse, What is sensor?-Definition from WhatIs.com, https://whatis.techtarget.com/definition/sensor, 2005.

[80] What is smart-sensor?-Definition from WhatIs.com, http://whatis.techtarget.com/definition/smart-sensor, 2016.

[81] C. Perera, A. Zaslavsky, P. Christen, and D. Georgakopoulos, Context aware computing for the Internet of Things: A survey, IEEE Communications Surveys Tutorials, vol. 16, no. 1, pp. 414-454, 2014.

[82] K. Rose, S. Eldridge, and L. Chapin, The Internet of Things: An overview, The Internet Society, vol. 80, pp. 1-53, 2015.

[83] M. Aazam and E. Huh, Fog computing and smart gatewaybased communication for cloud of things, in Proc. of 2014 International Conference on Future Internet of Things and Cloud, Barcelona, Spain, 2014, pp. 464-470.

[84] I. Davidson, Machine-to-machine (M2M) gateway: Trusted and connected intelligence-EE publishers, https://www.ee.co.za/article/machine-to-machine-m2mgateway-trusted-and-connected-intelligence.html, 2016. 


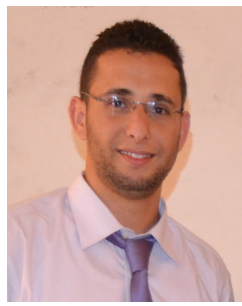

Mohammed Amine Bouras received the BS and MS degrees from the School of Computer Science, University of Laghouat, Algeria, in 2013 and 2015, respectively. $\mathrm{He}$ is currently a $\mathrm{PhD}$ candidate at the School of Computer and Communication Engineering, University of Science and Technology Beijing, China. He focuses on the identity management in healthcare and IoTs. His research interests include IoTs, blockchain, healthcare IT, and identity and access management.

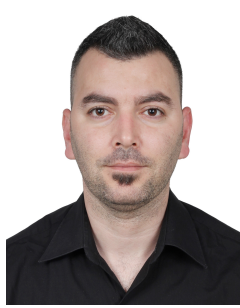

Fadi Farha received the BS degree from Informatics Engineering Faculty, Aleppo University, Syria in 2009. He received the MS degree from University of Science and Technology Beijing, China. He is currently working toward the $\mathrm{PhD}$ degree in the same school. His current research interests include Physical Uncolnable Function (PUF), smart home, security solutions, ZigBee, computer architecture, and hardware security.
Intelligent and Converged Networks, 2020, 1(1): 18-36

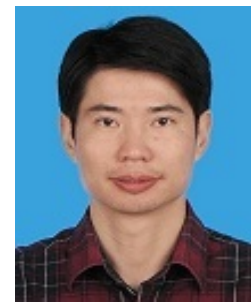

Huansheng Ning received the $\mathrm{PhD}$ degree from Beihang University in 2001. $\mathrm{He}$ is a professor and the vice dean of the School of Computer and Communication Engineering, University of Science and Technology Beijing, China. $\mathrm{He}$ is the founder of the Cyberspace and Cybermatics International Science and Technology Cooperation Base. He has published more than 170 journal/conference papers. His research interests include cybermatics, IoTs, and cyber-physical social systems. 